\title{
The first nucleophilic substitution reaction of organoindium reagents with sulfonyl chlorides: a facile method for preparation of vinyl sulfones
}

\author{
Guisheng Deng* and Jingyuan Zou
}

College of Chemistry and Chemical Engineering, Hunan Normal University, Changsha, Hunan 410081, People's Republic of China

Key Laboratory of Chemical Biology and Traditional Chinese Medicine Research (Ministry of Education of China), Hunan Normal University, Changsha, Hunan 410081, China

E-mail: gsdeng@hunnu.edu.cn

DOI: http://dx.doi.org/10.3998/ark.5550190.0011.214

\begin{abstract}
The first nucleophilic substitution reaction of organoindium with sulfonyl chlorides has been exploited. This reaction of vinylindium, derived from terminal alkynes via hydroindation, with sulfonyl chlorides in the presence of $\mathrm{Ag}_{2} \mathrm{O}$ provides access to vinyl sulfones. The vinyl sulfones were formed highly regio- and stereo-selectively with $E$ stereochemistry.
\end{abstract}

Keywords: Vinyl sulfones, terminal alkynes, vinylindium, indium trichloride

\section{Introduction}

Vinyl sulfones are widely used as building blocks in synthetic organic chemistry. For example, the chemical versatility of the sulfone moiety enable these compounds to undergo highly stereoselective nucleophilic conjugate additions and cycloadditions, ${ }^{1}$ cyclopropanation, ${ }^{2}$ epoxidation, ${ }^{3}$ palladium-catalyzed cascade arylation with aryl iodides, ${ }^{4}$ radical conjugate additions, ${ }^{5}$ and aza-Michael addition. ${ }^{6}$ Vinyl sulfones have been used for total synthesis of natural products. ${ }^{7}$ Furthermore, the sulfone group can be removed at the end of a synthetic sequence by a variety of reductive, alkylative or oxidative methods, where the sulfone moiety is replaced by a hydrogen, an alkyl or aryl group generated from a suitable organometallic crosscoupling reagent, or an oxygen function. ${ }^{8}$ Vinyl sulfones can be transformed to vinyl silanes and germanes, ${ }^{9}$ and vinyl sulfides. ${ }^{10}$ Furthermore, vinyl sulfones in medicinal chemistry represent a new class of inhibitors of such as cysteine proteases ${ }^{11,12}$, inducible VCAM-1 expression ${ }^{13}$ and depeptidyl peptidase I (DPPI) ${ }^{14}$ and transpeptidase ${ }^{15}$.

Due to their wide application, various methodologies for the synthesis of vinyl sulfones have been developed. Main ways to prepare vinyl sulfones include oxidation of the corresponding $\beta$ - 
arylthio systems and $\beta$-elimination, ${ }^{16}$ ionic or radical addition of $\mathrm{PhSO}_{2} \mathrm{X}(\mathrm{X}=\mathrm{Cl}, \mathrm{Br}, \mathrm{I}, \mathrm{SePh}$, etc.) to alkenes followed by $\beta$-elimination, ${ }^{17}$ or to alkynes,${ }^{18}$ Horner-Emmons reactions of a carbonyl compound and a sulfonyl-substituted phosphonate carbanion, ${ }^{19 a} \beta$-elimination of halosulfones, ${ }^{19 b, 19 \mathrm{c}}$ cerium(IV) ammonium nitrate mediated oxidative addition of sulfinate and iodine to alkenes, ${ }^{19 \mathrm{~d}}$ and the Mizoroki-Heck reaction of arylboronic acids with phenyl vinyl sulfones, ${ }^{19 \mathrm{e}}$ palladium-catalyzed cross-coupling reactions, ${ }^{20}$ cross-metathesis of vinyl sulfones with terminal olefins, ${ }^{21}$ and copper(II)-catalyzed Cross-coupling of organoboronic acids and sulfinate salts, ${ }^{22}$ one-pot reaction of sulfinic acid sodium salts and dibromides, ${ }^{23}$ CuI-catalyzed coupling reaction of vinyl bromides with sulfinic acid salts, ${ }^{24}$ or AIBN catalyzed addition of polystyrenesupported selenosulfonates to alkenes. ${ }^{25}$

In particular, the protocol for the preparation of vinyl sulfones by treating vinyl organometallic reagents with sulfonyl derivatives seems to be direct and efficient. Vinylzirconium, ${ }^{26}$ vinylaluminium, ${ }^{27}$ vinyltin ${ }^{28}$ and vinylmercury ${ }^{29}$ have been utilized for this purpose. Although these methods have been proven powerful in the synthesis of vinyl sulfones, they generally suffer from drawbacks such as sensitivity to air and water ${ }^{26,27}$ unavailability of vinyl organometallic reagents, ${ }^{26-29}$ and the necessity for transition metal catalysts ${ }^{28}$ or toxic reagents and unusual reaction conditions incompatible with various functional groups. ${ }^{29}$ Thus, the development of convenient and highly stereoselective methodologies for the synthesis of vinyl sulfones via vinyl organometallic reagents is of current interest.

\section{Results and Discussion}

Due to mild reaction activity, organoindium reagents such as vinylindium can tolerate a wide range of functional groups, and selectively undergo many reactions. ${ }^{30}$ They are readily available, relatively inert to air and water, and thermally stable and readily handled without special precautions. To our knowledge, nucleophilic substitution reactions of organoindium reagents with sulfonyl chlorides have never been investigated. Based on our aim, in theory, vinyl sulfones can be generated by nucleophilic substitution reaction of vinylindium with sulfonyl chlorides.

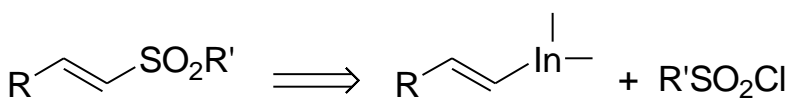

Vinylindium reagents were generated by hydroindation of terminal alkynes according to literature. ${ }^{31}$ We planned that resulting vinylindium reagents might react with sulfonyl chlorides without isolation. We initiated the investigations on this generation and substitution reaction of vinylindium by screening for optimal reaction conditions. Utilization of 0.1 equivalents of $\mathrm{InCl}_{3}$ and 1.5 equivalents of $\mathrm{NaBH}_{4}$ in hydroindation reaction of ethynylbenzene in $\mathrm{CH}_{3} \mathrm{CN}$, followed by treatment with tosyl chloride at room temperature, afforded an expected product $(E)-1-$ methyl-4-(styrylsulfonyl)benzene in less than 10\% yield (entry 1 in Table 1). This indicated that 
$\mathrm{InCl}_{3}$ did not work catalytically but a stoichiometric amount is necessary. When 1.5 equivalent of $\mathrm{InCl}_{3}$ was used in the hydroindation step under the same conditions, (E)-1-methyl-4(styrylsulfonyl)benzene was generated in up to $81 \%$ yield (entry 2 in Table 1). However, the expected product was afforded in only $40 \%$ yield when the substitution reaction of styrylindium intermediate with tosyl chloride was carried out at $0^{\circ} \mathrm{C}$ (entry 3 in Table 1). It was noted that a desired substitution reaction did not take place at $-15^{\circ} \mathrm{C}$ (entry 4 in Table 1), and 1-methyl-4(phenethylsulfonyl)benzene was generated in $46 \%$ yield via the reduction reaction of $(E)-1$ methyl-4-(styrylsulfonyl)benzene at $60^{\circ} \mathrm{C}$ (entry 5 in Table 1). When $\mathrm{CH}_{2} \mathrm{Cl}_{2}$ and EtOAc were used as solvent, yields were poor (entries 6 and 7 in Table 1). Especially, no desired reaction took place in benzene (entry 8 in Table 1). We could deduce no formation of vinylindium in benzene, as quenching with saturated $\mathrm{NH}_{4} \mathrm{Cl}$ previous to adding $\mathrm{TsCl}$ did not give styrene under the same conditions. Presumably, this reaction was a vinylindium formation-controlled process. However, in $\mathrm{InCl}_{3}-\mathrm{NaBH}_{4}-\mathrm{CH}_{3} \mathrm{CN}$ system, reaction velocity could obviously enhance in the presence of one equivalent of silver oxide (entry 9 in Table 1). This showed that silver oxide promotes the reaction of vinylindium intermediates with tosyl chloride. Therefore, the optimal reaction system was as follows: using 1.5 equivalents of $\mathrm{InCl}_{3}$ for the formation of vinylindium, $\mathrm{CH}_{3} \mathrm{CN}$ as solvent, one equivalent of $\mathrm{Ag}_{2} \mathrm{O}$ as additive, room temperature in the substitution step.

Table 1. Optimization of the reaction conditions for the generation of vinylindium intermediate and the formation of (E)-1-methyl-4-(styrylsulfonyl)benzene

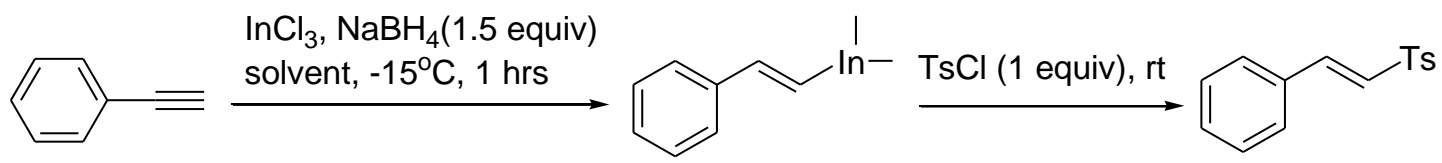

\begin{tabular}{ccccccc}
\hline Entry & $\begin{array}{c}\mathrm{InCl}_{3} \\
\text { (equiv) }\end{array}$ & Solvent & $\begin{array}{c}\text { Additive } \\
(1 \text { equiv) }\end{array}$ & $\begin{array}{c}\text { Reaction } \\
\text { temperature }\end{array}$ & $\begin{array}{c}\text { Reaction } \\
\text { time }(\mathrm{h})\end{array}$ & $\begin{array}{c}\text { Yield } \\
(\%)^{a}\end{array}$ \\
\hline 1 & 0.1 & $\mathrm{CH}_{3} \mathrm{CN}$ & no & $\mathrm{rt}$ & 15 & $<10$ \\
2 & 1.5 & $\mathrm{CH}_{3} \mathrm{CN}$ & no & $\mathrm{rt}$ & 15 & 81 \\
3 & 1.5 & $\mathrm{CH}_{3} \mathrm{CN}$ & no & $0^{\circ} \mathrm{C}$ & 15 & 40 \\
4 & 1.5 & $\mathrm{CH}_{3} \mathrm{CN}$ & no & $-15^{\circ} \mathrm{C}$ & 15 & $\mathrm{NR}^{c}$ \\
5 & 1.5 & $\mathrm{CH}_{3} \mathrm{CN}$ & no & $60^{\circ} \mathrm{C}$ & 15 & $36 \%{ }^{d}$ \\
6 & 1.5 & $\mathrm{CH}_{2} \mathrm{Cl} 2$ & no & $\mathrm{rt}$ & 15 & $20 \%$ \\
7 & 1.5 & $\mathrm{EtOAc}^{\circ}$ & no & $\mathrm{rt}$ & 15 & trace $^{2}$ \\
8 & 1.5 & benzene & no & $\mathrm{rt}$ & 15 & $\mathrm{NR}^{c}$ \\
9 & 1.5 & $\mathrm{CH}_{3} \mathrm{CN}$ & $\mathrm{Ag}_{2} \mathrm{O}$ & $\mathrm{rt}$ & 5 & 80 \\
\hline
\end{tabular}

${ }^{a}$ Isolated yields. ${ }^{b}$ Substitution reaction time. ${ }^{c}$ No reaction. ${ }^{d}$ By-product, 1-methyl-4(phenethylsulfonyl)benzene, was generated in $46 \%$ yield. 
Subsequently, in $\mathrm{InCl}_{3}-\mathrm{NaBH}_{4}-\mathrm{CH}_{3} \mathrm{CN}$ system, we examined terminal alkynes $\mathbf{1}$ and sulfonyl chlorides 2 in the reaction sequence in the presence of one equivalent of $\mathrm{Ag}_{2} \mathrm{O}$ (Scheme 1 ). The results were summarized in Table 2 . The results showed that $p$ - or $m$ - substituent on substituted phenyl rings, such as alkyl and halogen, had unfavorable effect on the yields when $p$ methylbenzenesulfonyl chloride was used as electrophile (entries 1-5 in Table 2). The effects of substituents on substituted phenyl rings were similar to that above-mentioned when benzenesulfonyl chloride and methanesulfonyl chloride were employed in preparation of vinyl sulfones (entries 6-9 in Table 2). In all case only $E$-isomer 3 was formed. But under the same conditions, the aliphatic terminal alkynes did not react at all (entries 10-12 in Table 2).

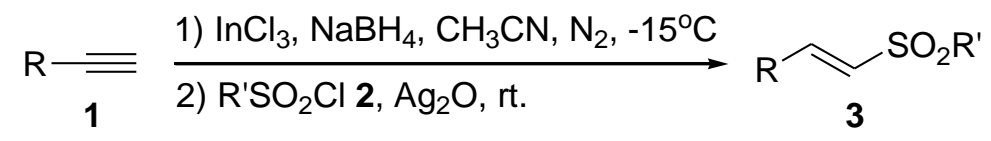

Scheme 1. Preparation of vinyl sulfones 3 via vinylindium intermediates.

Table 2. Preparation of vinyl sulfones $\mathbf{3}$ starting from alkynes $\mathbf{1}$ via vinylindium in the presence of one equivalent of $\mathrm{Ag}_{2} \mathrm{O}$

\begin{tabular}{|c|c|c|c|c|c|}
\hline Entry & $\mathrm{R}$ & $\mathrm{R}^{\prime}$ & $\begin{array}{c}\text { Reaction } \\
\text { time }(\mathrm{h})\end{array}$ & $\begin{array}{c}\text { Yield }^{a}(\%) \\
\text { of } \mathbf{3 a - i} \\
\end{array}$ & 3a-i \\
\hline 1 & & & 5 & 80 & $\mathbf{3 a}$ \\
\hline 2 & & & 10 & 63 & $3 b$ \\
\hline 3 & & & 15 & 36 & $3 c$ \\
\hline 4 & & & 15 & 66 & $3 d$ \\
\hline 5 & & & 24 & 46 & $3 \mathbf{e}$ \\
\hline 6 & & & 15 & 71 & 3f \\
\hline 7 & & & 24 & 47 & $3 g$ \\
\hline 8 & & $-\mathrm{CH}$ & 15 & 77 & $3 h$ \\
\hline 9 & & $-\mathrm{CH}$ & 15 & 70 & $3 \mathbf{i}$ \\
\hline
\end{tabular}


Table 2. Continued

\begin{tabular}{|c|c|c|c|c|c|}
\hline Entry & $\mathrm{R}$ & $\mathrm{R}^{\prime}$ & $\begin{array}{l}\text { Reaction } \\
\text { time }(\mathrm{h})\end{array}$ & $\begin{array}{c}\text { Yield }^{a}(\%) \\
\text { of } \mathbf{3 a - i}\end{array}$ & $3 a-\mathbf{i}$ \\
\hline 10 & $\mathrm{HOCH}_{2}-$ & & 15 & $\mathrm{NR}^{b}$ & \\
\hline 11 & $n-\mathrm{C}_{6} \mathrm{H}_{13}-$ & & 15 & $\mathrm{NR}^{b}$ & \\
\hline 12 & $n-\mathrm{C}_{4} \mathrm{H}_{9}-$ & & 15 & $\mathrm{NR}^{b}$ & \\
\hline
\end{tabular}

${ }^{a}$ Isolated yields. ${ }^{b}$ no reaction.

In most cases, the reaction of vinylindium with sulfonyl chlorides was favored by the presence of $\mathrm{Ag}_{2} \mathrm{O}$ as additive. A reasonable explanation is that $\mathrm{Ag}_{2} \mathrm{O}$ seems to promote the leave of chlorine as anion, while an attack by vinylindium is at the same time made in the opposite direction, thus leading to favorable generation of vinyl sulfones 3 (Scheme 2).

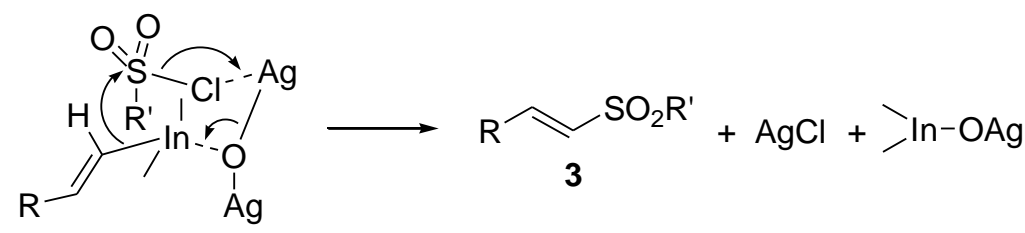

Scheme 2. A reasonable mechanism for the generation of vinyl sulfones $\mathbf{3}$ in the presence of $\mathrm{Ag}_{2} \mathrm{O}$.

\section{Conclusions}

In summary, nucleophilic substitution reaction of organoindium reagents with sulfonyl chlorides was investigated for the first time. This protocol proved to be a facile method for the synthesis of vinyl sulfones starting from terminal arynes via vinylindium. This new method has several advantages, such as mild reaction conditions, simple experimental process, highly regio- and stereoselectively. More efficient methods for the formation of vinylindium starting from other alkynes and potential uses of this methodology in preparation of other sulfones are under investigation in our laboratory.

\section{Experimental Section}

General. All solvents were dried before use. All chemical reagents were analytical grade and used without further purification. NMR spectra were recorded on a Bruker Avance DPX-500 
spectrometer in $\mathrm{CDCl}_{3}$ with tetramethylsilane as an internal standard. Infrared spectra were obtained using a Nicolet Nexus FT-IR spectrophotometer. Elemental analyses were obtained using a Thermofinnigan Flash-Ea 1112 series apparatus. MS (FAB) and HRMS spectra were recorded on waters ZQ2000 and Bruker APEX IV, respectively. Melting points were measured by an optical microscopic melting point instrument and are uncorrected. Reaction monitoring was accomplished by TLC on silica gel PolyGram SILG/UV 254 plates.

\section{Preparative procedure for vinyl sulfones}

A mixture of $\mathrm{InCl}_{3}(0.75 \mathrm{mmol})$ and $\mathrm{NaBH}_{4}(2.5 \mathrm{mmol})$ in dry $\mathrm{CH}_{3} \mathrm{CN}(3 \mathrm{ml})$ was stirred at -15 ${ }^{\circ} \mathrm{C}$ under nitrogen for $30 \mathrm{~min}$, and then terminal alkyne $(0.5 \mathrm{mmol})$ was added. After stirring for another hour, sulfonyl chloride $(0.5 \mathrm{mmol})$ dissolved in $\mathrm{CH}_{3} \mathrm{CN}(1 \mathrm{ml})$ was injected, followed by addition of $\mathrm{Ag}_{2} \mathrm{O}(0.5 \mathrm{mmol})$. The reaction mixture was warmed to room temperature and stirred for another 5-24 hours. The mixture was filtered and washed by $\mathrm{CH}_{3} \mathrm{CN}$ ( $3 \times 3 \mathrm{ml}$ ). Combined filtrate was concentrated in vacuum. The residue was purified by column chromatography on silica gel (100-200 mesh) by eluting with petroleum ether/ethyl acetate (6:1) to give the corresponding vinyl sulfones 3a-I (Table 2).

(E)-1-Methyl-4-(styrylsulfonyl)benzene (3a). Yield: 80\%; white solid (from hexane/EtOAc); mp 103-105 ${ }^{\circ} \mathrm{C}$ (lit. ${ }^{32} \mathrm{mp} 98-99{ }^{\circ} \mathrm{C}$ ); IR (KBr): $v_{\max }$ 1602, 1449, 1304, 1146, 974, 811, 747, 698, 601; ${ }^{1} \mathrm{H}$ NMR $\left(500 \mathrm{MHz}, \mathrm{CDCl}_{3}\right) \delta 2.43(\mathrm{~s}, 3 \mathrm{H}), 6.85(\mathrm{~d}, J=15.5 \mathrm{~Hz}, 1 \mathrm{H}), 7.34(\mathrm{~d}, J=8 \mathrm{~Hz}$, $2 \mathrm{H}), 7.38-7.41(\mathrm{~m}, 3 \mathrm{H}), 7.47(\mathrm{dd}, J=8,2 \mathrm{~Hz}, 2 \mathrm{H}), 7.65(\mathrm{~d}, J=15.5 \mathrm{~Hz}, 1 \mathrm{H}), 7.83$ (d, $J=8 \mathrm{~Hz}$, $2 \mathrm{H})$.

(E)-1-Methyl-4-(4-methylstyrylsulfonyl)benzene (3b). Yield: 63\%; white solid (from hexane/EtOAc); mp 117-119 ${ }^{\circ} \mathrm{C}$; IR (KBr): $v_{\max } 1610,1450,1306,1144,977,816,793,580 ;{ }^{1} \mathrm{H}$ NMR (500 MHz, $\left.\mathrm{CDCl}_{3}\right) \delta 2.37(\mathrm{~s}, 3 \mathrm{H}), 2.43(\mathrm{~s}, 3 \mathrm{H}), 6.79(\mathrm{~d}, J=15.5 \mathrm{~Hz}, 1 \mathrm{H}), 7.19$ (d, $J=8$ $\mathrm{Hz}, 2 \mathrm{H}), 7.34(\mathrm{~d}, J=8 \mathrm{~Hz}, 2 \mathrm{H}), 7.37(\mathrm{~d}, J=8 \mathrm{~Hz}, 2 \mathrm{H}), 7.63(\mathrm{~d}, J=15.5 \mathrm{~Hz}, 1 \mathrm{H}), 7.82(\mathrm{~d}, J=8$ $\mathrm{Hz}, 2 \mathrm{H}) .{ }^{13} \mathrm{C} \mathrm{NMR}\left(125.8 \mathrm{MHz}, \mathrm{CDCl}_{3}\right) \delta 144.23,142.01,141.70,138.03,129.92,129.78$, 129.76, 128.53, 127.66, 126.48, 21.59, 21.49. Anal. Calcd. for $\mathrm{C}_{16} \mathrm{H}_{16} \mathrm{O}_{2} \mathrm{~S}: \mathrm{C}, 70.56 ; \mathrm{H}, 5.92$. Found: C, 70.69; H, 5.90. MS (FAB) m/z: $295[\mathrm{M}+\mathrm{Na}]^{+}$. HRMS calcd for $\left[\mathrm{C}_{16} \mathrm{H}_{16} \mathrm{O}_{2} \mathrm{~S}+\mathrm{H}\right]$ : 273.0949, found: 272.0948 .

(E)-1-tert-Butyl-4-(2-tosylvinyl)benzene (3c). Yield: 36\%; white solid (from hexane/EtOAc); mp 77-79 ${ }^{\circ} \mathrm{C}$; IR (KBr): $v_{\max } 1616,1455,1308,1147,973,811,789,575 ;{ }^{1} \mathrm{H}$ NMR (500 MHz, $\left.\mathrm{CDCl}_{3}\right) \delta 1.31(\mathrm{~s}, 9 \mathrm{H}), 2.43(\mathrm{~s}, 3 \mathrm{H}), 6.80(\mathrm{~d}, J=15.5 \mathrm{~Hz}, 1 \mathrm{H}), 7.33(\mathrm{~d}, J=8 \mathrm{~Hz}, 2 \mathrm{H}), 7.40-7.43$ $(\mathrm{m}, 4 \mathrm{H}), 7.64(\mathrm{~d}, J=15.5 \mathrm{~Hz}, 1 \mathrm{H}), 7.82(\mathrm{~d}, J=8 \mathrm{~Hz}, 2 \mathrm{H}) ;{ }^{13} \mathrm{C} \mathrm{NMR}\left(125.8 \mathrm{MHz}, \mathrm{CDCl}_{3}\right) \delta$ $144.24,141.95,138.00,130.29$, 129.92, 128.41, 127.65, 126.61, 126.06, 125.06, 34.98, 31.10, 21.61. Anal. Calcd. for $\mathrm{C}_{19} \mathrm{H}_{22} \mathrm{O}_{2} \mathrm{~S}$ : C, 72.57; H, 7.05. Found: C, 72.50; H, 7.07. MS (FAB) $m / z$ : $337[\mathrm{M}+\mathrm{Na}]^{+}$. HRMS calcd for $\left[\mathrm{C}_{19} \mathrm{H}_{22} \mathrm{O}_{2} \mathrm{~S}+\mathrm{H}\right]: 315.1419$, found: 315.1419 .

(E)-1-Methyl-3-(2-tosylvinyl)benzene (3d). Yield: 66\%; white solid (from hexane/EtOAc); mp $82-83{ }^{\circ} \mathrm{C}$; IR (KBr): $v_{\max } 1610,1443,1304,1142,976,821,777,745,713,696,589 ;{ }^{1} \mathrm{H}$ NMR $\left(500 \mathrm{MHz}, \mathrm{CDCl}_{3}\right) \delta 2.35(\mathrm{~s}, 3 \mathrm{H}), 2.43(\mathrm{~s}, 3 \mathrm{H}), 6.83(\mathrm{~d}, J=15.5 \mathrm{~Hz}, 1 \mathrm{H}), 7.22-7.23(\mathrm{~m}, 1 \mathrm{H})$, 7.27-7.29 (m, 3H), $7.34(\mathrm{~d}, J=8 \mathrm{~Hz}, 2 \mathrm{H}), 7.62(\mathrm{~d}, J=15.5 \mathrm{~Hz}, 1 \mathrm{H}), 7.82(\mathrm{~d}, J=8 \mathrm{~Hz}, 2 \mathrm{H}) ;{ }^{13} \mathrm{C}$ 
NMR $\left(125.8 \mathrm{MHz}, \mathrm{CDCl}_{3}\right) \delta 144.33,142.15,138.83,137.86,132.42,131.95,129.96,129.11$, 128.95, 127.71, 127.37, 125.78, 21.62, 21.27. Anal. Calcd. for $\mathrm{C}_{16} \mathrm{H}_{16} \mathrm{O}_{2} \mathrm{~S}: \mathrm{C}, 70.56 ; \mathrm{H}, 5.92$. Found: C, 70.61; H, 5.89. MS (FAB) m/z: $295[\mathrm{M}+\mathrm{Na}]^{+}$. HRMS calcd for $\left[\mathrm{C}_{16} \mathrm{H}_{16} \mathrm{O}_{2} \mathrm{~S}+\mathrm{H}\right]$ : 273.0949, found: 273.0950 .

(E)-1-Chloro-3-(2-tosylvinyl)benzene (3e). Yield: 46\%; White solid (from hexane/EtOAc); mp 65-67 ${ }^{\circ} \mathrm{C}$; IR (KBr): $v_{\max } 1604,1468,1293,1143,981,838,774,694,602 ;{ }^{1} \mathrm{H}$ NMR (500 MHz, $\left.\mathrm{CDCl}_{3}\right) \delta 2.45(\mathrm{~s}, 3 \mathrm{H}), 6.86(\mathrm{~d}, J=15.5 \mathrm{~Hz}, 1 \mathrm{H}), 7.34-7.39(\mathrm{~m}, 5 \mathrm{H}), 7.45-7.46(\mathrm{~m}, 1 \mathrm{H}), 7.59(\mathrm{~d}$, $J=15.5 \mathrm{~Hz}, 1 \mathrm{H}), 7.82(\mathrm{~d}, J=8 \mathrm{~Hz}, 2 \mathrm{H}) ;{ }^{13} \mathrm{C} \mathrm{NMR}\left(125.8 \mathrm{MHz}, \mathrm{CDCl}_{3}\right) \delta 144.69,140.21$, 137.33, 135.13, 134.26, 130.97, 130.34, 130.08, 129.26, 128.17, 127.83, 126.77, 21.67. Anal. Calcd. for $\mathrm{C}_{15} \mathrm{H}_{13} \mathrm{ClO}_{2} \mathrm{~S}$ : C, 61.53; H, 4.48. Found: C, 61.60; H, 4.50. MS (FAB) m/z: 315 $[\mathrm{M}+\mathrm{Na}]^{+}$. HRMS calcd for $\left[\mathrm{C}_{15} \mathrm{H}_{13} \mathrm{ClO}_{2} \mathrm{~S}+\mathrm{H}\right]: 293.0403$, found: 293.0404 .

(E)-(2-(Phenylsulfonyl)vinyl)benzene (3f). Yield: 71\%; white solid (from hexane/EtOAc); mp 68-70 ${ }^{\circ} \mathrm{C}$ (lit. ${ }^{33} \mathrm{mp} \mathrm{70-71}{ }^{\circ} \mathrm{C}$ ); IR (KBr): $v_{\max } 1605,1446,1301,1142,969,743,687,591 ;{ }^{1} \mathrm{H}$ NMR (500 MHz, $\left.\mathrm{CDCl}_{3}\right) \delta 6.86(\mathrm{~d}, J=15.5 \mathrm{~Hz}, 1 \mathrm{H}), 7.39-7.42(\mathrm{~m}, 3 \mathrm{H}), 7.48-7.50(\mathrm{~m}, 2 \mathrm{H})$, 7.54-7.57 (m, 2H), 7.61-7.64 (m, 1H), 7.69 (d, $J=15.5 \mathrm{~Hz}, 1 \mathrm{H}), 7.95(\mathrm{~d}, J=8 \mathrm{~Hz}, 2 \mathrm{H})$.

(E)-1-Methyl-4-(2-(phenylsulfonyl)vinyl)benzene (3g). Yield: 47\%; white solid (from hexane/EtOAc); mp 136-138 ${ }^{\circ} \mathrm{C}$ (lit. ${ }^{34} \mathrm{mp} 139-140{ }^{\circ} \mathrm{C}$ ); IR (KBr): $v_{\max } 1605,1450,1309,1144$, 980, 828, 754, 686, 593; ${ }^{1} \mathrm{H}$ NMR $\left(500 \mathrm{MHz}, \mathrm{CDCl}_{3}\right) \delta 2.37(\mathrm{~s}, 3 \mathrm{H}), 6.80(\mathrm{~d}, J=15.5 \mathrm{~Hz}, 1 \mathrm{H})$, $7.19(\mathrm{~d}, J=8 \mathrm{~Hz}, 2 \mathrm{H}), 7.38(\mathrm{~d}, J=8 \mathrm{~Hz}, 2 \mathrm{H}), 7.53-7.63(\mathrm{~m}, 3 \mathrm{H}), 7.66(\mathrm{~d}, J=15.5 \mathrm{~Hz}, 1 \mathrm{H})$, $7.95(\mathrm{~d}, J=8 \mathrm{~Hz}, 2 \mathrm{H})$.

(E)-(2-(Methylsulfonyl)vinyl)benzene (3h). Yield: 77\%; yellow oil (lit. $\left.{ }^{35}\right)$; IR (KBr): $v_{\max } 1625$, 1453, 1306, 1124, 971, 753, 695, 586; ${ }^{1} \mathrm{H} \operatorname{NMR}\left(500 \mathrm{MHz}, \mathrm{CDCl}_{3}\right) \delta 3.04(\mathrm{~s}, 3 \mathrm{H}), 6.92(\mathrm{~d}, J=$ $15.5 \mathrm{~Hz}, 1 \mathrm{H}), 7.43-7.45(\mathrm{~m}, 3 \mathrm{H}), 7.52(\mathrm{dd}, J=7.5,1.5 \mathrm{~Hz}, 2 \mathrm{H}), 7.60(\mathrm{~d}, J=15.5 \mathrm{~Hz}, 1 \mathrm{H})$.

(E)-1-Methyl-4-(2-(methylsulfonyl)vinyl)benzene (3i). Yield: 70\%; yellow oil (lit. ${ }^{35}$ ); IR (KBr): $v_{\max } 1619,1450,1303,1134,974,810,595 ;{ }^{1} \mathrm{H} \mathrm{NMR}\left(500 \mathrm{MHz}, \mathrm{CDCl}_{3}\right) \delta 2.40(\mathrm{~s}, 3 \mathrm{H})$, $3.03(\mathrm{~s}, 3 \mathrm{H}), 6.86(\mathrm{~d}, J=15.5 \mathrm{~Hz}, 1 \mathrm{H}), 7.24(\mathrm{~d}, J=8 \mathrm{~Hz}, 2 \mathrm{H}), 7.41(\mathrm{~d}, J=8 \mathrm{~Hz}, 2 \mathrm{H}), 7.60(\mathrm{~d}, J$ $=15.5 \mathrm{~Hz}, 1 \mathrm{H})$.

\section{References}

1. For recent studies: (a) Zhu, Q.; Lu, Y. Org. Lett. 2009, 11, 1721. (b) Padmavathi, V.; Reddy, G. D.; Reddy, G. S. J. Heterocycl. Chem. 2008, 45, 1633. (c) Padmavathi, V.; Subbaiah, D. R. C. V.; Balaiah, A.; Reddy, B. C.; Padmaja, A. Indian J. of Chem., Section B: Org. Chem. Including Med. Chem. 2005, 44B, 2569. (d) Mauleon, P.; Alonso, I.; Rodriguez R. M.; Carretero, J. C. J. Org. Chem. 2007, 72, 9924. (e) Usera, A. R.; Posner, G. H. J. Org. Chem. 2007, 72, 2329. (f) Hernandez-Juan, F. A.; Xiong, X.; Brewer, S. E.; Buchanan, D. J.; Dixon, D. J. Synthesis 2005, 3283. (g) Grimaud, L.; Rotulo, D.; Perez, R. R.; Azam, L. G.; Prunet, J. Tetrahedron Lett. 2002, 43, 7477. (h) Fuchi, N.; Doi, T.; Harada, T.; Urban, J.; Cao, B.; Kahnc, M.; Takahashi, T. Tetrahedron Lett. 2001, 42, 1305. (i) Farthing, C. N.; Marsden, S. 
P. Tetrahedron Lett. 2000, 41, 4235. (j) Rincon, R.; Plumet, J. Synlett 2008, 911. (k) Mauleon, P.; Carretero, J. C. Chem. Commun. 2005, 4961. (1) Mauleon, P.; Carretero, J. C. Org. Lett. 2004, 6, 3195.

2. (a) Edwards, M. G.; Paxton, R. J.; Pugh, D. S.; Whitwood, A. C.; Taylor, R. J. K., Synthesis 2008, 3279. (b) Cao, W.; Zhang, H.; Chen, J.; Zhou, X.; Shao, M.; McMills, M. C. Tetrahedron 2007, 64, 163.

3. (a) Dorow, R. L.; Tymonko, S. A. Tetrahedron Lett. 2006, 47, 2493. (b) Lopez-Pedrosa, J.M.; Pitts, M. R.; Roberts, S. M.; Saminathan, S.; Whittall, J. Tetrahedron Lett. 2004, 45, 5073. (c) De la Pradilla, R. F.; Castellanos, A.; Fernandez, J.; Lorenzo, M.; Manzano, P.; Mendez, P.; Priego, J.; Viso, A. J. Org. Chem. 2006, 71, 1569.

4. Mauleon, P.; Nunez, A. A.; Alonso, I.; Carretero, J. C. Chem.-A Eur. J. 2003, 9, 1511.

5. Srikanth, G. S. C.; Castle, S. L. Tetrahedron 2005, 61, 10377.

6. Calata, C.; Pfund, E.; Lequeux, T. J. Org. Chem. 2009, 74, 9399.

7. (a) Piccardi, R.; Renaud, P. Eur. J. Org. Chem. 2007, 4752. (b) McLaughlin N. P.; Evans, P. J. Org. Chem. 2010, 75, 518.

8. For recent studies: (a) Wnuk, S. F.; Garcia, P. I. Jr.; Wang, Z. Org. Lett. 2004, 6, 2047. (b) Farhat, S.; Marek, I. Angew. Chem. Int. Ed. 2002, 41, 1410. (c) Yoshida, K.; Hayashi, T. J. Am. Chem. Soc. 2003, 125, 2872.

9. Wnuk, S. F.; Garcia, P. I. Jr.; Wang, Z. Org. Lett. 2004, 6, 2047.

10. Sacasa, P. R.; Zayas, J.; Wnuk, S. F. Tetrahedron Lett. 2009, $50,5424$.

11. Palmer, J. T.; Rasnick, D.; Klaus, J. L.; Brömme, D. J. Med. Chem. 1995, 38, 3193.

12. Santos, M. M. M.; Moreira, R. Mini-Reviews in Medicinal Chemistry 2007, 7, 1040.

13. Ni, L.; Zheng, X. S.; Somers, P. K.; Hoong, L. K.; Hill, R. R.; Marino, E. M.; Suen, K.-L.; Saxena, U.; Meng, C. Q. Bioorg. Med. Chem. Lett. 2003, 13, 745.

14. (a) Korver, G. E.; Kam, C.-M.; Powers, J. C.; Hudig, D. Int. J. Immunopharmacol. 2001, 1, 21. (b) Kam, C. M.; Götz, M. G.; Koot, G.; McGuire, M.; Thiele, D.; Hudig, D.; Powers, J. C. Arch. Biochem. Biophys. 2004, 427, 123.

15. Frankel, B. A.; Bentley, M.; Kruger, R. G.; McCafferty, D. G. J. Am. Chem. Soc. 2004, 126, 3404.

16. (a) Orita, A.; Katakami, M.; Yasui, Y.; Kurihara, A.; Otera, J. Green Chem. 2001, 3, 13. (b) Magnier, E.; Tordeux, M.; Goumont, R.; Magder, K.; Wakselman, C. J. Fluorine Chem. 2003, 124, 55.

17. For recent studies: (a) Nair, V.; Augustine, A.; Suja, T. D. Synthesis 2002, 2259. (b) Nair, V.; Augustine, A.; George, T. G.; Nair, L. G. Tetrahedron Lett. 2001, 42, 6763.

18. (a) Liu, X.; Duan, X.; Pan, Z.; Han, Y.; Liang, Y. Synlett 2005, 1752. (b) Edwards, G. L.; Muldoon, C. A.; Sinclair, D. J. Tetrahedron 1996, 52, 7779.

19. (a) Popoff, I. C.; Denver, J. L. J. Org. Chem. 1969, 34, 1128. (b) Sinnreich, J.; Asscher, M. J. Chem. Soc., Perkin Trans. 1 1972, 1543. (c) Hopkins, P. B.; Fuchs, P. L. J. Org. Chem. 1978, 43, 1208. (d) Nair, V.; Augustine, A.; George, T. G.; Nair, L. G. Tetrahedron Lett. 2001, 42, 6763. (e) Kabalka, G. W.; Guchhait, S. K. Tetrahedron Lett. 2004, 45, 4021. 
20. (a) Cacchi, S.; Fabrizi, G.; Goggiamani, A.; Parisi, L. M.; Bernini, R. J. Org. Chem. 2004, 69, 5608. (b) Kabalka, G. W.; Guchhait, S. K. Tetrahedron Lett. 2004, 45, 4021. (c) Bian, M,; Xu, F.; Ma, C. Synthesis 2007, 2951.

21. (a) Michrowska, A.; Bieniek, M.; Kim, M.; Klajn, R.; Grela, K. Tetrahedron 2003, 59, 4525.

(b) Grela, K.; Bieniek, M. Tetrahedron Lett. 2001, 42, 6425.

22. Huang, F.; Batey, R. A. Tetrahedron 2007, 63, 7667.

23. Guan, Z.-H.; Zuo, W.; Zhao, L.-B.; Ren, Z.-H.; Liang, Y.-M. Synthesis 2007, 1465.

24. Bao, W.; Wang, C. J. Chem. Res. 2006, 396.

25. Qian, H.; Huang, X. Synlett 2001, 1913.

26. Duan, D.-H.; Huang, X. Synlett 1999, 317.

27. (a) Signore, G.; Malanga, C.; Menicagli, R. Tetrahedron 2008, 64, 11218. (b) Signore, G.; Samaritani, S.; Malanga, C.; Menicagli, R. Synthesis 2006, 762. (c) Signore, G.; Calderisi, M.; Malanga, C.; Menicagli, R. Tetrahedron 2007, 63, 177.

28. Labadie, S. S. J. Org. Chem. 1989, 54, 2496.

29. Hershberger, J.; Russell, G. A. Synthesis 1980, 475.

30. For recent representative studies: (a) Nishimoto, Y.; Moritoh, R.; Yasuda, M.; Baba, A. Angew. Chem. Int. Ed. 2009, 48, 4577. (b) Black, D. A.; Arndtsen, B. A. Org. Lett. 2006, 8, 1991. (c) Baker, L.; Minehan, T. J. Org. Chem. 2004, 69, 3957. (d) Takami, K.; Yorimitsu, H.; Shinokubo, H.; Matsubara, S.; Oshima, K. Org. Lett. 2001, 3, 1997. (e) Font-Sanchis, E.; Céspedes-Guirao, F. J.; Sastre-Santos, Á.; Fernández-Lázaro, F. J. Org. Chem., 2007, 72, 3589. (f) Miao, W.; Lu, W.; Chan, T. H. J. Am. Chem. Soc. 2003, 125, 2412. (g) Lehmann, U.; Awasthi, S.; Minehan, T. Org. Lett. 2003, 5, 2405.

31. Xue, Z.; Yang, D. Y.; Wang, C. Y. J. Organomet. Chem. 2006, 691, 247.

32. Reeves, D. C.; Rodriguez, S.; Lee, H.; Haddad, N.; Krishnamurthy, D.; Senanayake, C. H. Tetrahedron Lett. 2009, 50, 2870.

33. Guan, Z.-H.; Zuo, W.; Zhao, L.-B.; Ren, Z.-H.; Liang, Y.-M. Synthesis 2007, 10, 1465.

34. Huang, X.; Duan, D.; Zheng, W. J. Org. Chem. 2003, 68, 1958.

35. Battace, A.; Zair, T.; Doucet, H.; Santelli, M. Synthesis 2006, 3495. 